\title{
Polymer-Based Photonic Crystals Fabricated with Single-Step Electron-Beam Lithography**
}

\author{
By Cen-Shawn Wu, Chih-Fu Lin, Hung-Yi Lin, Cho-Lun Lee, and Chii-Dong Chen*
}

Photonic crystals (PCs) are a novel optical nanostructure with periodic refractive-index modulation. They can give rise to a photonic band gap within which the propagation and emission of light are prohibited. The band gap can be tuned by geometric parameters such as the lattice constant or filling ratio to alter the transmission spectra or the dispersion of light propagating through the PC structure. $2 \mathrm{D} \mathrm{PCs},{ }^{[1-4]}$ which can manipulate the propagation of in-plane light at telecommunication wavelengths, ${ }^{[1,3]}$ have received much attention recently. Such a device consists of a 2D array of air holes embedded in a high-dielectric-constant slab constructed on top of a lowdielectric-constant substrate and can be made using presentday semiconductor fabrication technologies. However 2D PCs suffer from difficulties arising from unwanted coupling to free-space modes in the substrate. This coupling breaks translation symmetry and has to be avoided by carefully designing the crystal parameters. One of the common approaches to eliminating this coupling is to suspend the slabs using a $\mathrm{SiO}_{2}$ sacrificial layer, forming a quasi-3D PC structure. Silicon-oninsulator (SOI)-based suspended $2 \mathrm{D} \mathrm{PCs}{ }^{[5]}$ are a good example, in which the incident light is confined in the third dimension. But full confinement of photons can only be achieved in 3D PCs, in which a photonic band gap is present in all three directions and complete control of light propagation and

[*] Dr. C.-D. Chen, C.-L. Lee

Institute of Physics, Academia Sinica

Nankang, 115 Taipei, Taiwan (ROC)

E-mail: chiidong@phys.sinica.edu.tw

Dr. C.-D. Chen

Department of Electrical Engineering, National Chung Hsing

University

Taichung 402, Taiwan (ROC)

Dr. C.-S. Wu, Dr. C.-F. Lin

Graduate Institute of Electro-Optical Engineering and Department

of Electrical Engineering, National Taiwan University

Taipei 106, Taiwan (ROC)

Dr. C.-S. Wu

Institute of Physics, Academia Sinica

Nankang, 115, Taipei, Taiwan (ROC)

Dr. H.-Y. Lin

Department of Material Science, National University of Tainan

Tainan 700, Taiwan (ROC)

[***] We would like to thank Y. C. Tsai for fruitful discussions about the 3D PC band gap. This work is supported by the National Science Council No. NSC 95-2112-M-001-062-MY3. The fabrication work was performed at the Core-facility Center, Academia Sinica. emission is possible. Early work on 3D PCs utilized techniques such as self-assembly, ${ }^{[6]} 3 \mathrm{D}$ interference lithography, ${ }^{[7,8]}$ direct laser writing, ${ }^{[9,10]}$ glancing-angle deposition, ${ }^{[11]}$ and electron-beam lithography (EBL) with wafer fusion. ${ }^{[12]}$ However unsolved issues in these techniques still exist. For example, EBL with wafer fusion has layer-to-layer alignment problems, and both glancing-angle deposition and self-assembly techniques present difficulties for making defect modes such as waveguides or microcavities.

A polymer-based $\mathrm{PC}$ with a photonic band gap is a candidate for optical integrated circuits because of its low propagation loss at telecommunication wavelengths and compatibility with optical fibers. ${ }^{[13]}$ The photonic-band-structure calculation $^{[14]}$ has shown a complete band gap for transverse-electric (TE)-like modes. Furthermore, conjugated polymer ${ }^{[15-16]}$ has been shown to exhibit excellent performance in luminescence and lasing, and is thus an ideal building block for active optical components. In this paper, we demonstrate a simple new method to fabricate polymer-based suspended quasi-3D photonic slabs and 3D PCs.

Polymethylmethacrylate (PMMA) is a widely used polymer material because it is a standard resist for EBL and it can also be used in nanoimprint lithography ${ }^{[17]}$ owing to its low glasstransition temperature $\left(T_{g}=105^{\circ} \mathrm{C}\right)$. For PC applications, the PMMA layer should have good verticality, large aspect ratio, and high dimensional accuracy. In view of this, high-energy EBL is the most suitable technique because it has several advantages such as: 1) a long penetration depth into the resist with a small forward scattering angle, which is important for the thick-resist process; and 2) a negligible proximity effect due to a wide secondary-electron spread in the substrate (and thus negligible secondary-electron-induced exposure), which helps to reduce pattern broadening. Additional information about high energy EBL is given in Section 1 of the Supporting Information.

For the fabrication of a polymer-suspended PC structure with sub-100 $\mathrm{nm}$ resolution, the undercut of the sacrificial layer should be accurately controlled. To this end, we chose to use a PMMA/LOR bilayer polymer system because LOR (an abbreviation of "lift-off resist") is a polydimethylglutarimidebased polymer and has a much higher charge sensitivity than that of a top PMMA layer. Figure 1 shows a cross-section SEM image of a bilayer polymer after electron-beam exposure and development. In Figures S2-4 of the Supporting Information, we show that the size of undercut increases with development time for the bottom LOR layer. With a prolonged development time, the LOR layer can be removed 


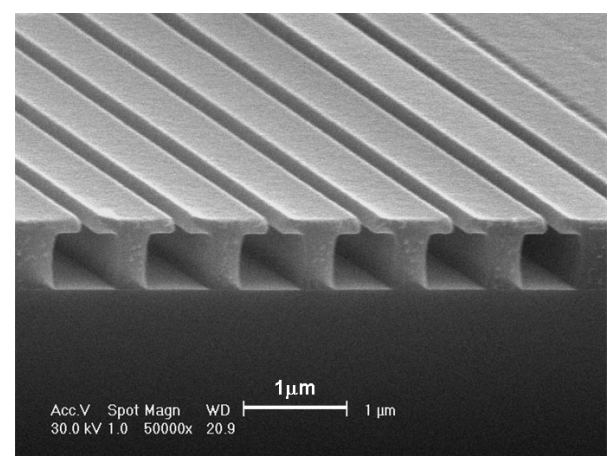

Figure 1. Cross-section SEM images of a T-shaped bilayer polymer. The roof layer and the post of "T" are made of PMMA and LOR, respectively. The gap in the PMMA layer is $100 \mathrm{~nm}$, and the undercut in the LOR layer is $300 \mathrm{~nm}$.

completely and the top PMMA becomes suspended. Figure 2 shows an SEM image of a suspended-polymer PC slab with a hexagonal array of air holes, in which the LOR layer served as a sacrificial layer. Suspended-polymer PC slabs were reported previously ${ }^{[18]}$ but in that case $\mathrm{SiO}_{2}$ substrates were used as the sacrificial layer, and a complicated fabrication process was employed. Another important feature of our bilayer polymer process is that the suspended structures can be made on virtually any kind of substrate, which is not possible in the case of semiconductor-based quasi-3D PCs.

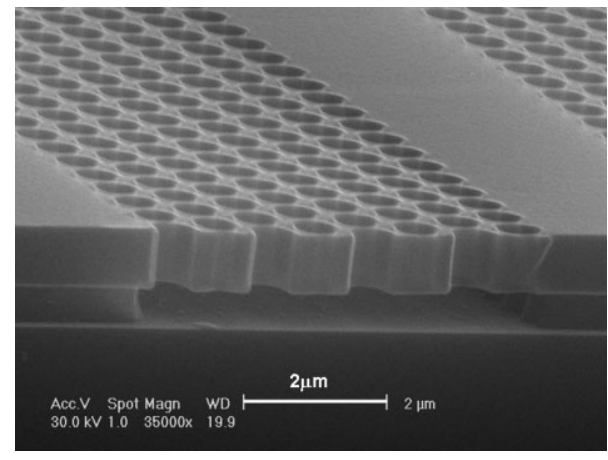

Figure 2. SEM image of a suspended PMMA quasi-3D PC slab with a hexagonal array of air holes. The hole radii are $260 \mathrm{~nm}$, and the lattice constant is $800 \mathrm{~nm}$.

A photonic-band-structure calculation ${ }^{[14]}$ has shown that suspended PCs possess a complete band gap for TE-like modes. Here we show that for a set of appropriate lattice parameters, the suspended PC slab can exhibit simultaneously a band gap for both TE-like modes and transverse magnetic (TM)-like modes at a $1.3 \mu \mathrm{m}$ wavelength. This calculation was performed using the plane-wave expansion method and index guiding in the third dimension. ${ }^{[19]}$ Although the slab is inherently a 2D PC, we constructed a quasi-3D PC structure by introducing sufficiently thick air layers on the top and bottom sides of the slab. In the calculation, a refractive index of 1.49 for PMMA and a domain size of $7 \times 11$ unit cells were used. Figure $3 \mathrm{a}$ and $\mathrm{b}$ show respectively the spectrum for TE-like modes and TM-like modes calculated using a slab thickness of $1.5 a$ and a air hole radius of $0.325 a$, where the lattice constant $a$ is assumed to be $800 \mathrm{~nm}$. The vertical axis is the frequency normalized to $2 \pi c / a$, where $c$ is the light speed in a vacuum. The radiation modes coupled to the free space are located above the light line (indicated by the solid curves). The inset indicates the points of high symmetry in the first Brillouin zone of the hexagon lattice. As indicated in Figure 3, the suspended PMMA PC slab exhibits two photonic band gaps for TE-like modes in wavelength ranges of 1356-1379 $\mathrm{nm}$ and 1290-1311 nm, and for TM-likes modes in wavelength ranges of 1538-1568 nm and 1212-1311 nm. The suspended PC slab thus exhibits photonic band gaps for both TE-like and TM-like modes at telecommunication wavelengths.
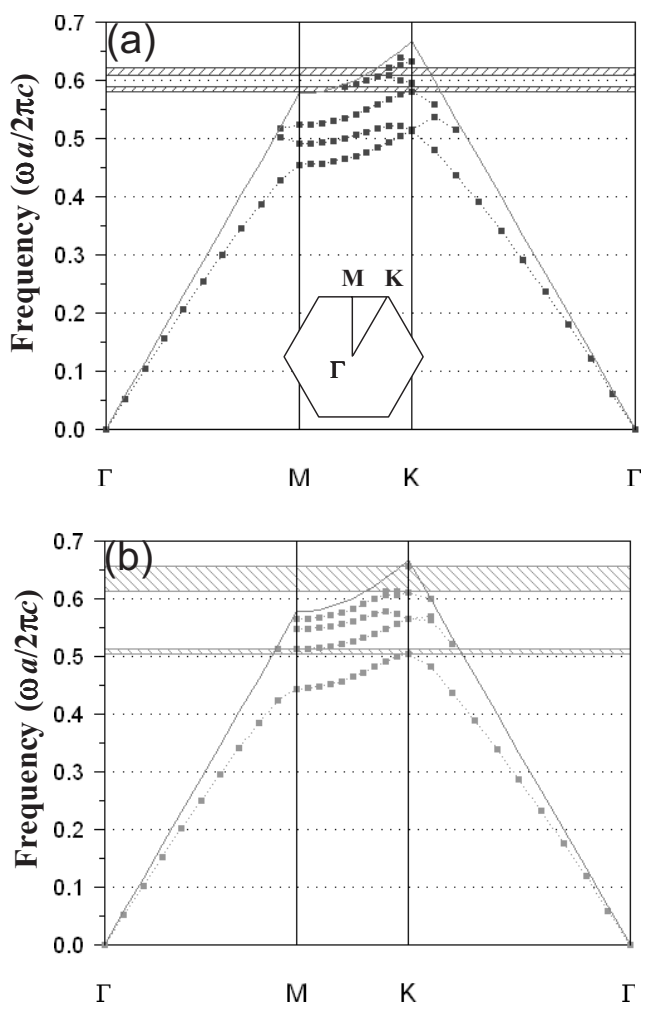

Figure 3. Photonic band structure of the suspended PMMA PC slab for a) TE-like modes and b) TM-like modes at $1.3 \mu \mathrm{m}$ wavelength. The inset indicates the points of high symmetry in the first Brillouin zone of the hexagon lattice.

With this technique, the fabrication of hexagonal arrays with small lattice constants becomes feasible and thus quasi3D PCs in the visible range can be realized. In Figure 4 color images of 5 samples taken under identical optical conditions are shown. The PC parameters of these samples, including the hole radius, lattice constant, and calculated band gap are listed in Table 1. Sample \#1 had a band gap in the red-light range, and hence showed a complementary sky-blue color. A 
Table 1. PC parameters of the five samples shown in Figure 4.The calculated PC band gaps are for TM-like modes.

\begin{tabular}{lccccc}
\hline & Sample \#1 & Sample \#2 & Sample \#3 & Sample \#4 & Sample \#5 \\
\hline Hole radius [nm] & 130 & 130 & 50 & 50 & 50 \\
Lattice constant [nm] & 400 & random & 400 & 350 & 300 \\
calculated & $605-635$ & & $605-34$ & $530-555$ & $454-484$ \\
band gap [nm] & & & & $583-593$ & $491-508$ \\
measured & & none & 660 & $445[1]$ & 460 \\
minimum & & & 527 & 524 \\
transmission wavelength [nm] & & & 650 & \\
\hline
\end{tabular}

[1] Not present in the calculation.
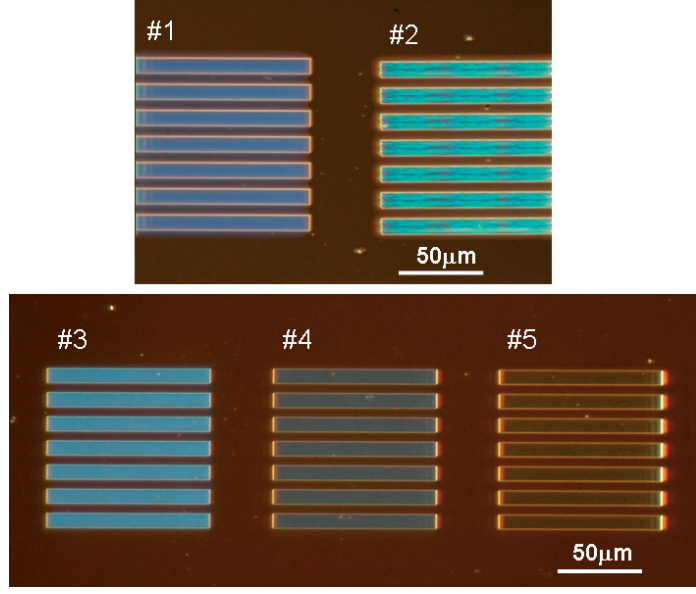

Figure 4. Color images of the suspended polymer PC slabs under irradiation of white light at a $45^{\circ}$ incident angle. Each sample contains 7 PC slabs, and measures $75 \mu \mathrm{m} \times 75 \mu \mathrm{m}$ in total area. Except sample \#2, which has a random lattice constant and does not possess a PC band gap, the other 4 samples show complementary colors of the corresponding band gaps.
Figure 4. Small deviations from the calculated values can possibly be attributed to slight deformations of the polymer structures, corrections in the reflective index of the polymers, and imperfection in the collimation of the incident beam. In addition, the transmission spectrum for a sample with a random distribution of $50 \mathrm{~nm}$ holes is displayed in Figure 5d as a control, which shows no particular absorption band. Figure 5d signifies that the absorptions in Figure $5 \mathrm{a}-\mathrm{c}$ are indeed due to the photonic band gaps, and not due to the polymer itself or air-hole scattering. This demonstrates the applicability of PMMA suspended slabs as quasi-3D PCs in the visible spectrum.

Although quasi-3D PC slabs are useful for specific applications, they have limited potential in applications requiring confinement in all directions, which can only be achieved with 3D PCs. Fabrication of 3D PCs for short-wavelength applications presents a great challenge even for present-day semiconductor fabrication technology as it requires definition and alignment of submicron features in the vertical. With long penetration depth in resists provided by the high-energy electron beam and the large contrast in exposure sensitivity between PMMA and LOR, we were able to make multilayer structures. This approach fully utilizes the self-alignment feature and requires only a single lithography step. Figure $6 a$ and $b$ show, respectively, the cross-section view of a ten-layer line-array structure and the top view of a ten-layer pillar structure made using a layer-by-layer spin-coating technique. It is worth noting that because of electron-beam forward scattering in the thick polymer, the diameter of the LOR posts at the bottom is somewhat narrower than that of the different color was shown by sample \#2, a control sample, which contained aperiodically distributed holes with hole radii and area density the same as those in sample \#1. The light-blue color is attributed to blue-dominated Rayleigh scattering because the hole size is close to the blue-light wavelength. The difference in colors of the scattered light between these two samples is evidence of a PC band gap in sample \#1. In order to show the effect of the PC band gap, we further tested three samples with the same air-hole radius of $50 \mathrm{~nm}$ but with different lattice constants ranging between 300 and $400 \mathrm{~nm}$; these samples displayed complementary colors in respect to their PC band gaps, namely, sky blue, royal blue, and amber as we expected.

The transmission spectrums for samples \#3-5 with a hole radius of $50 \mathrm{~nm}$ are displayed in Figure $5 \mathrm{a}-\mathrm{c}$, in which the calculated band gaps are shown in Table 1 for comparison. Reasonable agreement between the calculated and measured data support the microscope observations shown in
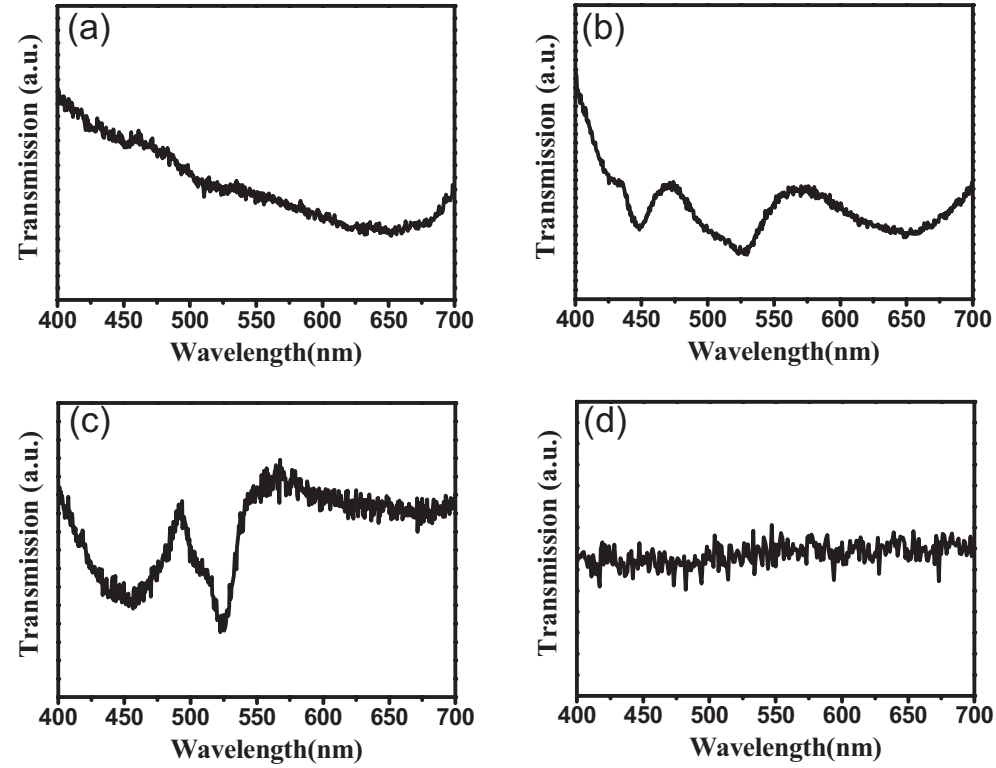

Figure 5. Transmission spectra of quasi-3D PC samples with varying lattice constants: a) $400 \mathrm{~nm}$, b) $350 \mathrm{~nm}$, and c) $300 \mathrm{~nm}$. The spectrum in (d) is for a sample with a random distribution of holes. 

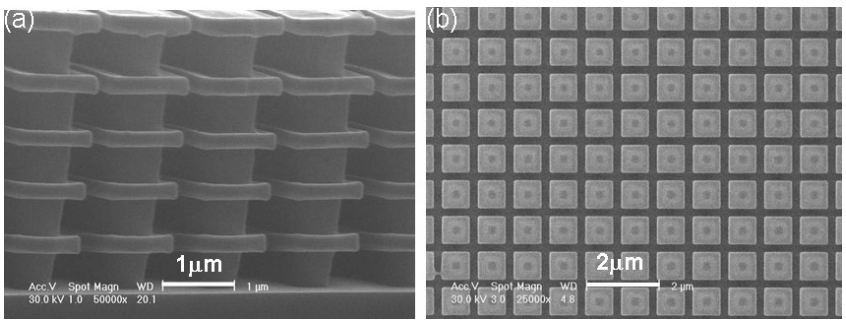

Figure 6. a) Cross-sectional view of a ten-layer line-array structure. b) Top view of a 3D PMMA/LOR pillar structure. The square PMMA roofs are $770 \mathrm{~nm}$ on each side and $200 \mathrm{~nm}$ in thickness. The LOR pillars are $550 \mathrm{~nm}$ on each side and $500 \mathrm{~nm}$ in height. The inter-roof distance is $230 \mathrm{~nm}$ and the lattice constant is $1 \mu \mathrm{m}$.

top ones. Because the top few layers have been developed nine times more than that of the lower layers, the small variation in feature sizes also suggests that the process window is wide regarding the developing time. The multilayer pillar structure shown in Figure $6 \mathrm{~b}$ has a tetragonal lattice symmetry with a basis consisting of a square "roof" supported by a square "pole", and a PC band gap is possible. Furthermore, inverted 3D pillars made of high-reflective-index dielectric materials would help in enlarging the local band gaps, making overlap between gaps more possible. The 3D pillars can be constructed by filling (e.g. electroplating ${ }^{[4]}$ ) the air gap using the multilayer polymer structure as a mold.

Defects are a prerequisite for PCs to function as light guides, switches or photon emitters/traps. In our approach, point or line defects can be made at locations beneath the air gaps by simply controlling the exposure dose. A demonstration of this concept, in which an under-exposure dose was applied to all line patterns except the specified one, is shown in Figure 7.

In conclusion, by making use of the large contrast in exposure sensitivities of PMMA and LOR, we were able to make suspended PMMA quasi-3D PC slabs with hexagonal arrays of air holes. The band gap calculation for this structure shows the existence of in-plane photonic band gaps in both TE-like and TM-like modes at telecommunication wavelengths. In addition, we demonstrated the operation of suspended PMMA

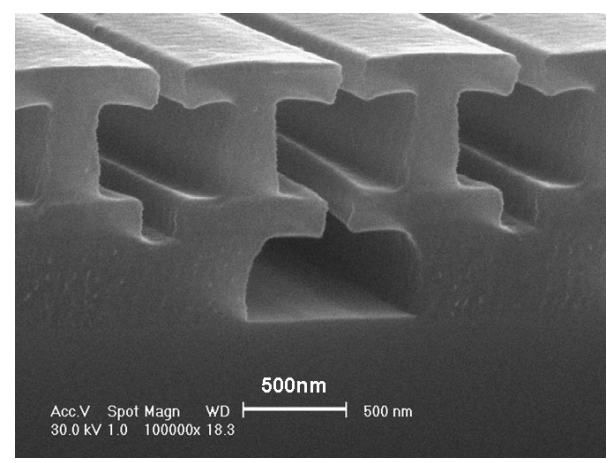

Figure 7. SEM image of a line defect created by adjusting the exposure dose.
quasi-3D PC slabs in the visible spectrum. Furthermore, we showed a simple new method for the fabrication of polymerbased 3D PCs, which will be useful for the implementation of low-cost and low-loss photonic band gap optical devices.

\section{Experimental}

Fabrication of Suspended Quasi-3D Polymer PCs: LOR (MicroChem Inc, LOR5A) was first spun at $4000 \mathrm{rpm}$ for $35 \mathrm{~s}$ and baked at $180^{\circ} \mathrm{C}$ for $5 \mathrm{~min}$ to form a $0.5 \mu \mathrm{m}$ thick polymer layer. PMMA (MicroChem Inc, PMMA4A) was then spin-coated at $4000 \mathrm{rpm}$ for $35 \mathrm{~s}$. This was followed by a soft bake at $135^{\circ} \mathrm{C}$ for $1 \mathrm{~h}$ to form a $0.2 \mu \mathrm{m}$ thick PMMA layer on top of the LOR. The bilayer polymers were then subjected to $100 \mathrm{keV}$ electron-beam exposures (Elionix Inc., ELS-7000). The top layer PMMA in the exposed area was then etched away by immersing the substrate in methylisobutylketone/isopropyl alcohol developer for $5 \mathrm{~s}$. This was followed by development of the bottom-layer LOR in tetramethyl-ammonium hydroxide (TMAH $2.2 \%$ ) for $45 \mathrm{~s}$. In this way, an undercut structure was readily produced. To fabricate suspended PCs, the top PMMA layer was made much thicker $(1.2 \mu \mathrm{m})$ so that it could be self-sustained, and the undercut was developed to extend over the entire LOR layer.

Fabrication of Multilayer Polymer Structures: The fabrication procedures are as follows: First, spin coating and baking of LOR and PMMA were carried out sequentially and repeatedly to form a superstructure containing 5 tiers of PMMA/LOR bilayers. The superstructure was then exposed with line-array or $2 \mathrm{D}$ mesh patterns using a $100 \mathrm{keV}$ electron-beam writer. The development process was then carried out layer-by-layer with appropriate developing times, starting from the top layer of the superstructure. Again, a methylisobutylketone/isopropyl alcohol mixture and TMAH $(2.2 \%)$ severed as developers for PMMA and LOR, respectively.

Optical Measurement Setup for Suspended Quasi-3D Polymer PC: Suspended PMMA PC slabs with lattice dimensions designed for a visible-light band gap were constructed on glass substrates and tested by using two methods. The results are shown in Figure 4 and Figure 5 , respectively. In the first approach, the samples were examined using an inverted microscope (Olympus IX71 with 40× objective lens) under dark-field illumination conditions; the scattered light was recorded with a color charge-coupled device (CCD) camera (Nikon D70). The slabs were illuminated by a light incident at $45^{\circ}$ from an incandescent filament such that the incident light propagated in the slabs along the $\Gamma$-K direction. In the second approach, a collimated monochromatic visible-light beam from a Xenon light source (Spectral products AST-XE-175-EX) was employed as the source, and the intensity of the transmitted light was measured using a photomultiplier tube (Hamamatsu H9305). The incident beam was also along the $\Gamma-\mathrm{K}$ direction of the slabs. The outlet light intensity was divided by the intensity of a nearby region without PC.

Received: October 31, 2006 Revised: March 8, 2007 Published online: August 31, 2007

[1] S. Noda, A. Chutinan, M. Imada, Nature 2000, 407, 608.

[2] H. G. Park, S. H. Kim, S. H. Kwon, Y. G. Ju, J. K. Yang, J. H. Baek, S. B. Kim, Y. H. Lee, Science 2004, 305, 1444.

[3] B. S. Song, S. Noda, T. Asano, Science 2003, 300, 1537.

[4] Y. W. Su, C. S. Wu, C. C. Chen, C. D. Chen, Advanced Materials $\mathbf{2 0 0 3}, 15,49$

[5] S. Takayama, H. Kitagawa, Y. Tanaka, T. Asano, S. Noda, Appl. Phys. Lett. 2005, 87, 061107.

[6] Y. A. Vlasov, X-Z. Bo, J. G. Strum, D. J. Norris, Nature 2001, 414, 289.

[7] M. Campbell, D. N. Sharp, M. T. Harrison, R. G. Denning, A. J. Tuberfield, Nature 2000, 404, 53. 
[8] J. S. King, E. Graugnard, O. M. Roche, D. N. Sharp, J. Scrimgeour, R. G. Denning, A. J. Turberfield, C. J. Summers, Adv. Mat. 2006, 18, 1561.

[9] K. K. Seet, V. Mizeikis, S. Juodkazis, H. Misawa, Appl. Phys. Lett. 2006, 88, 221101

[10] J. Serbin, M. Gu, Adv. Mat. 2006, 18, 221.

[11] S. R. Kennedy, M. J. Brett, O. Toader, S. John, Nano Lett. 2002, 2, 59.

[12] M. Qi, E. Lidorikis, Peter T. Rakich, S. G. Johnson, J. D. Joannopoulos, E. P. Ippen, H. I. Smith, Nature 2004, 429, 538.

[13] G. Böttger, C. Liguda, M. Schmidt, M. Eich, Appl. Phys. Lett. 2002, $81,2517$.

[14] C. S. Kee, S. P. Han, K. B. Yoon, C. G. Choi, H. K. Sung, S. S. Oh, H. Y. Park, S. Park, H. Schift, Appl. Phys. Lett. 2005, 86, 051101.
[15] R. H. Friend, R. W. Gymer, A. B. Holmes, J. H. Burroughes, R. N. Marks, C. Taliani, D. D. C. Bradley, D. A. Dos Santos, J. L. Brédas, M. Lögdlund, W. R. Salaneck, Nature 1999, 397, 121.

[16] F. Hide, M. A. Díaz-García, B. J. Schwartz, M. R. Andersson, Q. Pei, A. J. Heeger, Science 1996, 273, 1833.

[17] "Alternate Lithography: Unleashing the Potentials of Nanotechnology" (Ed. C. M. Sotomayor Torres), Kluwer Academic, Plenum Publishers, New York 2004

[18] R. R. Panepucci, B. H. Kim, V. R. Almeida, M. D. Jones, J. Vac. Sci. Technol. B 2004, 22, 3348.

[19] S. G. Johnson, S. Fan, P. R. Villeneuve, J. D. Joannopoulos, Phys. Rev. B: Condens. Matter Mater. Phys. 1999, 60, 5751. 